\title{
A GENERALIZATION OF COMMUTATIVE AND ALTERNATIVE RINGS. IV
}

\author{
ERWIN KLEINFELD ${ }^{1}$
}

ABSTRACT. We have shown previously for rings $R$ of characteristic $\neq 2,3$ which satisfy the three identities (i) $\left(x, y^{2}, x\right)=y \circ(x, y, x)$, (ii) $(x, y, z)+(y, z, x)+(z, x, y)=0$, and (iii) $((x, y), x, x)=0$, where $(a, b, c)=(a b) c-a(b c),(a, b)=a b-b a$, and $a \circ b=a b+b a$, that under the assumption of no divisors of zero, all such $R$ must be either associative or commutative. Here we weaken the Lie-admissible identity (ii) by assuming instead (iv) Lie-admissibility for every subring generated by two el ements. It turns out that rings without divisors of zero and of characteristic $\neq 2,3$ which satisfy (i), (iii) and (iv) are either commutative or alternative. If $S$ is a ring in which every subring generated by two elements is either commutative or associative, then identities (i), (iii) and (iv) hold in $S$, so that this result applies to $S$.

Main section. Identities (i), (iii) and (iv) hold in all commutative rings. They also hold in all alternative rings. Identities (i) and (iv) hold in Lie rings as well. One could of course weaken (iii) to the identity $(v)((x, y), x, x)^{2}$ $=0$, thus affording us a set of identities, namely (i), (iv) and (v), which are valid in all Lie rings as well and imply the same main result, but since (v) quickly implies (iii) under the hypothesis of no divisors of zero, this seems too artificial. However we contend that (i), (iii) and (iv) is a set of identities comparable to the noncommutative Jordan identities and worthy of consideration.

From now on we concentrate on the proof of the main theorem. We assume that $R$ represents a ring that satisfies identities (i), (iii) and (iv), has characteristic $\neq 2,3$ and has no divisors of zero.

Lemma 1. Every subring of $R$ which can be generated by two elements is either associative or commutative.

Received by the editors June 5, 1973.

AMS (MOS) subject classifications (1970). Primary 17A30, 17E05; Secondary $17 \mathrm{~A} 15,17 \mathrm{C} 05,17 \mathrm{D} 05$.

Key words and phrases. Commutative, alternative, flexible, noncommutative, Jordan, zero divisor.

1 This research was supported in part by the National Science Foundation under GP-32898 XI. 
Proof. Every subring which can be generated by two elements satisfies the identities (i), (ii) and (iii) and so the main result of [1] applies. This completes the proof of the lemma.

Lemma 2. $R$ satisfies the identity

$$
(a, b)(a, a, b)=0
$$

and its linearizations

$$
\begin{array}{r}
(a, b)(a, a, c)+(a, c)(a, a, b)=0 \\
(d, b)(a, a, b)+(a, b)(d, a, b)+(a, b)(a, d, b)=0,
\end{array}
$$

and

$$
\begin{aligned}
(d, b)(a, a, c) & +(a, b)(d, a, c)+(a, b)(a, d, c) \\
& +(d, c)(a, a, b)+(a, c)(d, a, b)+(a, c)(a, d, b)=0 .
\end{aligned}
$$

Proof. That (1) is an identity follows from the conclusion of Lemma 1. Identities (2) and (3) follow from linearizations of (1), while (4) can be obtained by linearizing either (2) or (3). This completes the proof of the lemma.

Main Theorem. $R$ is either commutative or alternative.

Proof. It follows from Lemma 1 that $R$ is flexible, that is that the identity

$$
(a, b, a)=0,
$$

holds, since both commutative and as sociative rings are flexible. If $R$ were also left alternative then $R$ would be alternative and we would be done. Hence we assume that $R$ is not left alternative. This implies the existence of elements $x$ and $y$ in $R$ such that $(x, x, y) \neq 0$. Again because of Lemma 1 this means that

$$
(x, y)=0 .
$$

Substitution of $a=x, b=y, c=r$ in (2) leads to

$$
(x, y)(x, x, r)+(x, r)(x, x, y)=0=(x, r)(x, x, y),
$$

because of (6). Thus using no divisors of zero we obtain

$$
(x, R)=0 .
$$

Substitution of $a=x, b=y, d=s$ in (3) leads to

$$
(s, y)(x, x, y)+(x, y)(s, x, y)+(x, y)(x, s, y)=0=(s, y)(x, x, y),
$$


because of (6). Thus using no divisors of zero we obtain

$$
(R, y)=0 .
$$

Substitution of $a=x, b=y, c=r, d=s$ in (4) leads to

$$
\begin{aligned}
(s, y)(x, x, r) & +(x, y)(s, x, r)+(x, y)(x, s, r)+(s, r)(x, x, y) \\
& +(x, r)(s, x, y)+(x, r)(x, s, y)=0=(s, r)(x, x, y),
\end{aligned}
$$

as a result of (7) and (8). But then using no divisors of zero once again we obtain

$$
(R, R)=0 .
$$

But (9) implies that $R$ is commutative. Thus we have shown that if $R$ is not alternative then it must be commutative. This completes the proof of the Theorem.

\section{REFERENCE}

1. Erwin Kleinfeld, A generalization of commutative and associative rings, Pacific J. Math. 38 ( 1971 ), 95-101. MR 46 \#5411.

DEPARTMENT OF MATHEMATICS, UNIVERSITY OF IOWA, IOWA CITY, IOWA 52242 